Penser les épidémies depuis la Chine, le Japon et la Corée

\title{
Le retour des dispositifs de protection anciens dans la gestion politique des épidémies
}

\section{Patrice Bourdelais}

\section{(2) OpenEdition Journals}

Édition électronique

URL : http://journals.openedition.org/extremeorient/345

DOI : 10.4000/extremeorient.345

ISSN : 2108-7105

Éditeur

Presses universitaires de Vincennes

Édition imprimée

Date de publication : 1 septembre 2014

Pagination : 241-246

ISBN : 978-2-84292-411-9

ISSN : 0754-5010

Référence électronique

Patrice Bourdelais, «Le retour des dispositifs de protection anciens dans la gestion politique des épidémies », Extrême-Orient Extrême-Occident [En ligne], 37 | 2014, mis en ligne le 01 septembre 2017, consulté le 23 avril 2019. URL : http://journals.openedition.org/extremeorient/345 ; DOI : 10.4000/ extremeorient.345 


\title{
Le retour des dispositifs de protection anciens dans la gestion politique des épidémies
}

\author{
Patrice Bourdelais
}

La lecture des textes rassemblés ici conduit tout d'abord au plaisir de la découverte de nouveaux aspects et résultats de la recherche sur des pays, époques et situations qui demeurent, quoi que l'on fasse, quelque peu lointains dans notre culture d'historien des épidémies et de la santé publique dans les pays d'Europe de l'Ouest. Chemin faisant dans le dossier, les rapprochements avec des situations européennes viennent à l'esprit, qu'il s'agisse de l'intervention forte du pouvoir royal coréen dans la deuxième moitié du XvIII siècle afin de maîtriser une épidémie de rougeole très agressive, de la découverte épidémiologique qui inverse les termes de la responsabilité dans la progression de la peste ou bien encore des liens qui unissent la révolution culturelle chinoise et une épidémie de méningite cérébro-spinale.

On peut y observer, comme dans l'Europe de l'Ouest, l'évolution de l'organisation de la lutte contre les épidémies par les États, qui peut être lue à la lumière des concepts de biopolitique et de gouvernementalité de Michel Foucault. L'exemple de la mise en place de mesures très contraignantes et précises par le roi Jeongjo en Corée offre une illustration de l'intervention de la Couronne afin de protéger et de faire fructifier la population et de renforcer les structures idéologiques traditionnelles de l'État. Celui de la dynamique entre la Révolution culturelle chinoise et l'épidémie de méningite cérébro-spinale met en scène les différentes phases de conciliation entre des gouvernementalités différentes: la raison d'État, dominante, et la santé publique, secondaire certes pendant quelques mois mais dont le caractère indispensable est intégré ensuite très rapidement à la raison d'État.

Chaque contribution à ce volume suscite la comparaison avec d'autres époques, d'autres épidémies et d'autres situations. Mais je m'arrêterai volontiers sur le cas de Taiwan qui est ici l'objet de deux articles assez complémentaires. Celui de Vincent Rollet analyse le lien entre la gestion des épidémies et l'approche sécuritaire qui s'est particulièrement manifestée lors 
de la gestion de l'épidémie de SRAS et de H5N1 en 2003-2005. À partir des travaux de Barry Buzan et al. (1998) et de la contribution de Sara Davies «Securitizing Infectious disease» (2008), il examine le processus par lequel ces maladies sont devenues des questions de sécurité nationale à Taiwan, en mettant l'accent sur les initiateurs de cette dynamique, leurs discours puis la façon dont se concrétise une telle approche. Les autorités taiwanaises ont décidé de considérer la présence du SRAS puis du H5N1 comme des questions de sécurité nationale qui se traduisent par une succession de mesures. Dès le lendemain de l'annonce du premier cas de SRAS, le Président de Taiwan déclare que la lutte contre la nouvelle épidémie a la même urgence que la défense de la sécurité nationale en général. Ce sont les citoyens et la stabilité économique du pays qui constituent l'objectif principal des mesures prises, y compris celles qui doivent éviter les comportements paniques. Cette initiative est considérée comme sans précédent. Bien entendu, un budget spécifique et très important est voté, il dépasse de beaucoup celui consacré à la lutte contre le VIH/Sida. Une quarantaine est organisée pour toute personne ayant été en contact avec un malade afin de prévenir une extension de la contagion. L'isolement était néanmoins plus ou moins strict, allant de l'enfermement complet à la possibilité de quitter la quarantaine pour acheter par exemple ses repas. Les personnes qui ne se soumettent pas aux règles de la quarantaine encourent aussi bien des amendes élevées (286 personnes concernées) que des peines de prison. Plus d'un millier de soldats sont aussi mobilisés, dont certains chargés d'opérations de désinfection. Le choix d'un hôpital militaire afin d'accueillir et de traiter les malades, ainsi que les communiqués quotidiens des autorités sanitaires, ne peuvent que renforcer le sentiment que l'on mène une véritable guerre contre l'ennemi.

Lorsque l'épidémie de H5N1 menace, fin 2005, l'État réagit de la même manière (réunion du haut conseil de sécurité nationale, vote d'un budget spécifique élevé). La présence de volailles vivantes sur les marchés est interdite. Taiwan envisage de produire, à des fins d'usage domestique, du Tamiflu, avant d'avoir reçu l'autorisation des laboratoires Roche, ce qui correspond à une décision exceptionnelle qui n'est finalement pas mise en exécution car le H5N1 ne se manifeste pas sur l'île.

Le caractère spectaculaire des mesures prises, en particulier la mise en place de quarantaines, lors du SRAS paraît avoir frappé les esprits des contemporains par leur nouveauté. Il suffit pourtant de retourner cinquante ans en arrière pour constater la banalité de ce dispositif à Taiwan même. Dans les conditions épidémiologiques de sortie de la Seconde Guerre mondiale, face à la multiplication des cas de malaria mais aussi de peste et de choléra, on retrouve alors ce qui avait constitué la base des dispositifs de protection des 
épidémies depuis six siècles au moins. Avec le retour de Taiwan à la Chine en 1945, les épidémies importées du continent se multiplient: en particulier peste, choléra, variole et une recrudescence de la malaria. C'est alors que sont mis en place des contrôles (épaulés par des examens de laboratoires) et quarantaines dans les ports taiwanais afin d'éviter les importations de Chine continentale. Les contrôles sont à nouveau renforcés en 1947 (afin de répondre au niveau d'exigence international des dispositifs de quarantaines) si bien que toute importation d'épidémie de Chine continentale est évitée au cours de l'année suivante. À partir de 1949, l'aide médicale et sanitaire américaine se retire de Chine continentale et se concentre sur Taiwan, contribuant en peu d'années à réduire la malaria et à supprimer la peste, le choléra et la variole. La mémoire de cette période aurait-elle disparu de Taiwan au début des années 2000 ?

Pour les historiens de la longue durée, le grand virage de la fin du $\mathrm{xx}^{\mathrm{e}}$ siècle, du côté des organisations, est lié au constat d'un échec: en dépit des progrès de la science et de la hausse du niveau de vie, les épidémies, strictement nouvelles ou ré-émergentes, deviennent à nouveau des dangers pour chaque pays ainsi que pour la prospérité et la stabilité mondiales. Dans son article, Michael Shiyung Liu cite deux travaux qui concluent à l'efficacité forte des quarantaines mises en place à la sortie de la guerre. Vincent Rollet cite lui aussi une estimation de la réduction de la contagion et du nombre de décès réalisée grâce aux mesures de confinement et de quarantaine lors de l'épidémie de SRAS. Pourquoi s'étonner de tels succès? Comment expliquerait-on, si la quarantaine n'était pas efficace, qu'elle ait été une pratique systématique en Europe et au-delà du milieu du XIv ${ }^{\mathrm{e}}$ au milieu du XIX ${ }^{\mathrm{e}}$ siècle (Bourdelais 2003)? L'historiographie s'est souvent complue à souligner les failles de tout système coercitif, mais l'irruption de la peste n'a été qu'exceptionnelle par rapport aux risques que les ports d'Europe occidentale encouraient chaque année. Dans plusieurs cas bien documentés, le dispositif de cordons sanitaires et de quarantaines a permis de sauver des régions entières de l'épidémie (je pense à l'arrêt de la peste du début du règne de Louis XIV stoppée par le cordon protecteur tendu entre Rouen et Reims afin de sauver la Capitale, puis à la Peste de Marseille qui, en 1720-1722, ne réussit guère à sortir de la Provence et d'une partie du Languedoc). Lorsque les États ont délibérément organisé des cordons sanitaires et des quarantaines, les épidémies ont la plupart du temps été stoppées. Mais ces dispositifs avaient un coût élevé. Économique tout d'abord, car non seulement ils interrompaient les relations commerciales pendant des mois, voire des années, mais ils nécessitaient le maintien sous les armes de dizaines de milliers de soldats. Politique ensuite, car ils entravaient la libre circulation des individus qui, lorsqu'ils tentaient de traverser les lignes, risquaient d'être abattus par un soldat (Baldwin 1999). 
La conciliation entre les contraintes liées au maintien de la santé publique et la liberté individuelle fut toujours un sujet délicat, au point que le cordon sanitaire et la quarantaine furent au $\mathrm{XIX}^{\mathrm{e}}$ siècle synonymes de régimes totalitaires. L'Angleterre libérale proposa alors un nouveau régime de protection, fondé sur l'examen médical à l'arrivée des bateaux, l'hospitalisation des malades dans des hôpitaux dédiés et le suivi pendant quelques semaines des passagers qui paraissaient bien portants. C'est à cette époque que la responsabilité individuelle du malade qui fréquentait des lieux ou des transports publics fut engagée; elle pouvait le conduire à devoir payer une amende ou à effectuer quelques jours de prison. Mais il ne faut pas oublier qu'au XIX ${ }^{\mathrm{e}}$ siècle, et parce que la plupart des épidémies suivaient l'itinéraire des cargos de l'est vers l'ouest de la Méditerranée, l'Angleterre avait réussi à sécuriser le bassin occidental en contrôlant les passages à Malte puis à Gibraltar. Il devenait alors moins risqué de lever les dispositifs contraignants à l'entrée en Angleterre! Les Commissions internationales sanitaires furent chargées, jusque dans les années 1930, de continuer à protéger l'Europe de l'Ouest des épidémies en déplaçant la charge du contrôle à Istanbul et Alexandrie (Roemer 1994).

C'est précisément ce qui n'est plus possible depuis les années 1980 du fait de la globalisation qui s'amplifie régulièrement : toujours plus d'avions, emmenant toujours plus de voyageurs directement d'un point du globe à un autre, y compris dans des régions reculées ou récemment ouvertes au contact. Cette mobilité sans précédent ne permet plus aucun contrôle réel. Il s'agit de la principale raison de l'inquiétude des milieux sanitaires internationaux qui scrutent avec vigilance - il s'agit bien de leur fonction - l'émergence d'une nouvelle épidémie toujours possible et, devrait-on dire, attendue. Tous les gouvernants du monde savent à quel point une épidémie grave peut déstabiliser profondément leur société ; cela explique bien, aussi, le virage des années 1990-2000.

L'horizon historique d'éradication des maladies infectieuses, que les pays développés pensaient pouvoir un jour atteindre, s'est éloigné (Cockburn 1963). Les nouvelles découvertes sur les logiques du vivant ont montré qu'il est vain de préparer des vaccins antiviraux trop à l'avance. La globalisation, la faiblesse des antiviraux disponibles et les résistances de plus en plus nombreuses aux antibiotiques convainquent de la grande fragilité de notre système de santé globale actuel. Pour les épidémiologistes, toutes les variables présentent des valeurs qui doivent conduire à la catastrophe que l'on attend, avec crainte, mais aussi avec un soupçon d'intérêt car il sera alors temps de passer à l'action, de mettre en place tous les plans qui ont été préparés (parfois répétés) depuis des années. De ce fait, une tendance à la surestimation du risque est aujourd'hui probablement présente dans la majorité des milieux responsables du contrôle de la santé publique internationale. 
Mais la tonalité actuelle des travaux qui parlent de sécurisation des maladies infectieuses laisse penser que leurs auteurs ont oublié qu'il ne s'agit en rien d'une nouveauté (mis à part le rôle nouveau de l'OMS qui accentue, avec de nouveaux moyens, le rôle tenu pendant longtemps par les Conférences sanitaires internationales) mais simplement du retour à une attitude de très longue durée des États qui se défendent tout autant contre les grands dangers épidémiques que contre le voisin hostile. Souvent d'ailleurs, la contagion vient bien de l'étranger, comme l'ennemi. La contribution de William Johnston sur les réactions face aux premières épidémies de choléra au Japon au XIX $x^{\mathrm{e}}$ siècle le montre. A contrario, le mouvement hygiéniste, qui prend son essor dans les pays d'Europe occidentale au XVIII et s'exprime complètement au XIX ${ }^{e}$ siècle, consiste à rompre avec cette tradition et à considérer que la maladie ne vient pas de l'étranger mais qu'elle est bien présente au cœur des villes et des villages, liée aux conditions de salubrité très insuffisantes pour assurer un environnement qui ne soit pas propice à la multiplication des maladies sur place. Cette inversion de conception conduit à faire circuler l'air afin qu'il ne stagne pas (dans les logements et les immeubles par exemple) et elle aboutit au modèle anglais du lavage permanent de la ville qui doit recevoir beaucoup d'eau afin qu'elle en draine les effluents vers la rivière voisine. Cette grande circulation hydrique a constitué le modèle de référence de l'hygiène publique moderne, elle supposait l'adduction d'eau courante et la construction de réseaux d'égouts (Hamlin 1998). À partir du moment où le choléra devient endémique au Japon, les conditions d'une prise en charge de l'organisation de la salubrité sont d'ailleurs réunies. Mais cette lecture comparée ne peut se faire qu'en creux car l'axe des travaux présentés ici ne concerne pas les transferts sanitaires de l'Occident vers l'Orient sauf en ce qui concerne la Chine des années 1930 et les grands acteurs de l'influence médicale américaine. Finalement, les effets des oppositions politiques, voire guerrières, entre Chine du Nord et Chine du Sud, entre Japon, Chine et Corée, entre Japon, Chine et Taiwan sur les représentations sanitaires et sur les politiques de protection contre les épidémies, y sont très présents. C'est une dimension qui paraît, du fait des contributions rassemblées ici, plus discriminante qu'en Europe. 
Patrice Bourdelais

\section{BIBLIOGRAPHIE}

Baldwin Peter (1999). Contagion and the State in Europe, 1830-1930. Cambridge, Cambridge University Press.

Bourdelais Patrice (2003). Les Épidémies terrassées. Une histoire de pays riches. Paris, Éditions de La Martinière.

Buzan, BarRy, Waever Ole, De Wilde Jaap (1998). Security: A New Framework for Analysis. Londres, Lynne Rienner Publishers.

CockBuRn Aidan (1963). The Evolution and Eradication of Infectious Diseases. Baltimore, Johns Hopkins Press.

DAVIES Sara (2008). «Securizing Infectious Disease». International Affairs, vol. 84, 2 : 296-313 (Wiley Online Library).

Hamlin Christopher (1998). Public Health and Social Justice in the Age of Chadwick: Britain, 1800-1854. Cambridge, Cambridge University Press.

RoEmer Milton (1994). «Internationalism in Medicine and Public Health». In Dorothy Porter, The History of Public Health and the Modern State. Amsterdam/Altanta, Rodopi : 403-423. 